\section{Testing a Theory-Based Model of Suicidality in a Community Sample}

\author{
Rui C. Campos' and Ronald R. Holden ${ }^{2}$
}

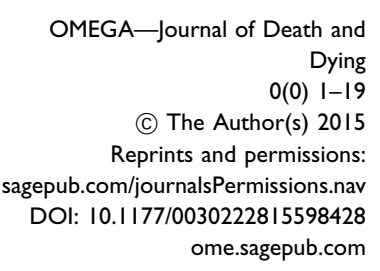

@SAGE

\begin{abstract}
The aim of the present study is to test a theory-based model of suicide in a low-risk nonclinical sample. A community sample of convenience of 200 adults, 102 men and 98 women, responded to the Depressive Experiences Questionnaire, the Center for the Epidemiologic Studies of Depression Scale, the Psychache Scale, the Interpersonal Needs Questionnaire, and the Suicide Behaviors Questionnaire Revised. The hypothesized structural equation model, including trait dimensions of self-criticism and neediness, and state dimensions of depression, psychache, perceived burdensomeness, and thwarted belongingness, fit the observed data well and significantly explained $49 \%$ of the variance of suicidality.
\end{abstract}

\title{
Keywords
}

suicidality, self-criticism, neediness, depression, psychache, perceived burdensomeness, thwarted belongingness

Suicide is an important mental health issue. The World Health Organization (2012) estimates that every year almost one million people die by suicide worldwide and that nonfatal suicidal behaviors - such as suicide attempts and self-directed violence-have estimated rates 10 to 20 times higher than death by suicide.

\footnotetext{
'Departamento de Psicologia, University of Évora, Portugal

${ }^{2}$ Department of Psychology, Queen's University, Kingston, ON, Canada

\section{Corresponding Author:}

Rui C. Campos, Departamento de Psicologia, Escola de Ciências Sociais, Universidade de Évora, Apartado 94, Évora 7002 -554, Portugal.

Email: rcampos@uevora.pt
} 